\title{
Collision Cross Sections in Terms of Local Observables*
}

\author{
H. ARAKI** and R. HAAG \\ Department of Physics, University of Illinois \\ Urbana, Illinois
}

Received July 1, 1966

\begin{abstract}
Asymptotic relations for matrix elements of quasilocal operators are given which generalize and extend the Lehmann-Symanzik-Zimmermann relations. These relations allow the simulation of a coincidence arrangement of particle detectors in the mathematical frame of the theory and thereby the expression of collision cross sections in terms of expectation values of observables.
\end{abstract}

\section{Introduction}

Within the framework of Quantum Field Theory particle collisions have always been treated by means of formulas and algorithms which are based on the asymptotic relation I below. Denoting the vacuum state by $|o\rangle$, the state of a single particle of type $i$ and momentum $\mathbf{k}$ by $|\mathbf{k}, i\rangle^{1}$ with the normalization

we have the

$$
\left\langle\mathbf{k}^{\prime}, j \mid \mathbf{k}, i\right\rangle=\delta_{i j} \delta^{3}\left(\mathbf{k}^{\prime}-\mathbf{k}\right)
$$

Asymptotic relation $I$ :

If $Q$ is an arbitrary quasilocal ${ }^{2}$ operator with $\langle o|Q| o\rangle=o$ and if the point $x$ moves to infinity in a time-like direction ${ }^{3}$ then, for $x_{0} \rightarrow+\infty$

$$
Q(x) \rightarrow \sum_{i} \int d^{3} k\left(\langle\mathbf{k}, i|Q(x)| o\rangle a_{i}^{\dagger \text { out }}(\mathbf{k})+\langle o|Q(x)| \mathbf{k}, i\rangle a_{i}^{\text {out }}(\mathbf{k})\right)
$$

and for $x_{0} \rightarrow-\infty$

$$
Q(x) \rightarrow \sum_{i} \int d^{3} k\left(\langle k, i|Q(x)| o\rangle a_{i}^{\dagger \mathrm{in}}(\mathbf{k})+\langle o|Q(x)| \mathbf{k}, i\rangle a_{i}^{\text {in }}(\mathbf{k})\right) .
$$

* This paper results from the collaboration of the authors during the winter semester $63 / 64$ at Urbana, Illinois and was partly supported by the NSF.

** Present address: Research Institute for Mathematical Sciences, Kyoto University, Kyoto, Japan.

${ }^{1}$ If the particle has spin we shall, for simplicity, consider here the description of the spin orientation included in the index $i$.

${ }^{2}$ For a definition of "quasilocal" see the beginning of section II.

3 We use small Latin letters to denote 4 -vectors, boldface letters for 3 -vectors. Thus $x=\left(\mathbf{x}, x_{0}\right)$ denotes a point in space-time with the time component $x_{0}$ and space components $\mathbf{x}$. The energy-momentum 4-vector of a particle of type $i$ is written correspondingly as $k=\left(\mathbf{k}, k_{0}\right)$ where, of course, $k_{0}=\left(\mathbf{k}^{2}+m_{i}^{2}\right)^{1 / 2}$ and $m_{i}$ is the particle mass. For the Lorentz scalar product we write $k x=\mathbf{k x}-k_{0} x_{0}$.

6 Commun. math. Phys., Vol. 4 
Here $Q(x)$ is defined as the translate of $Q$ by the 4 -vector $x$;

$$
Q(x)=U(x) Q U^{-1}(x)
$$

where $U(x)$ is the translation operator through $x$. The operators $a_{i}^{\text {† out }}(\mathbf{k})$, $a_{i}^{\text {out }}(\mathbf{k})$ are the usual creation and destruction operators for an outgoing particle of type $i$ and momentum $\mathbf{k}$. Their commutation relations correspond to the normalization convention (1); if $Q$ is an ordinary quasilocal operator one has

$$
\left[a_{i}^{\text {out }}(\mathbf{k}), a_{j}^{\text {out }}\left(\mathbf{k}^{\prime}\right)\right]=\delta_{i j} \delta^{3}\left(\mathbf{k}-\mathbf{k}^{\prime}\right),
$$

if $Q$ is a quasilocal Fermi operator ${ }^{4}$ one has

$$
\left\{a_{i}^{\text {out }}(\mathbf{k}), a_{j}^{\dagger \text { out }}\left(\mathbf{k}^{\prime}\right)\right\}=\delta_{i j} \delta^{3}\left(\mathbf{k}-\mathbf{k}^{\prime}\right)
$$

where \{\} denotes the anticommutator. One has to understand the limit relations (2), (3) in the following sense. They are valid only for matrix elements between fixed, normalizable state vectors $\Psi, \Phi$ of which at least one must lie in a certain dense domain (weak convergence). It is easily seen that the matrix elements of the right hand side vanish like $x_{0}^{-3 / 2}$ as $x_{0} \rightarrow \infty$. Therefore the exact content of equation (2) is that the difference between the left hand side and the right hand side has matrix elements which vanish faster than $x_{0}^{-3 / 2}$.

We may call (2), (3) the generalized LSZ-relations [1]. To see the connection with the formulas of [1] one uses the fact that the $x$-dependence on the right hand side is explicitly known:

$$
\langle\mathbf{k}, i|Q(x)| o\rangle=e^{-i k x} F_{i}(\mathbf{k}) ;\langle o|Q(x)| \mathbf{k}, i\rangle=e^{i k x} G_{i}(\mathbf{k}) .
$$

If we multiply (2) with a function $f(x)$ which is a positive frequency solution of the Klein-Gordon equation to mass $m$, i.e.

$$
f(x)=(2 \pi)^{-3 / 2} \int f(\mathbf{k}) e^{i\left(\mathbf{k} \mathbf{x}-\sqrt{k^{2}+m^{2}} x_{0}\right)} d^{3} k
$$

we get after integrating over 3 -dimensional space

$$
\begin{gathered}
\int_{x_{0}=t} Q(x) f(x) d^{3} x \underset{t \rightarrow+\infty}{\longrightarrow}(2 \pi)^{+3 / 2} \sum_{j} \int d^{3} k\left(F_{j}(\mathbf{k}) f(\mathbf{k}) e^{i\left(\sqrt{\mathbf{k}^{2}+m_{i}^{2}}-\sqrt{\left.\mathbf{k}^{2}+m^{2}\right) t}\right.}\right. \\
a_{j}^{\dagger \text { out }}(\mathbf{k})+G_{j}(\mathbf{k}) f(-\mathbf{k}) e^{-i\left(\sqrt{\mathbf{k}^{2}+m_{i}^{2}}+\sqrt{\left.\mathbf{k}^{2}+m^{2}\right) t} a_{j}^{\text {out }}(\mathbf{k})\right) .}
\end{gathered}
$$

If $F_{i}, G_{i}, f,\left\langle\Phi\left|a_{i}^{\text {tout }}(k)\right| \Psi\right\rangle,\left\langle\Phi\left|a_{i}^{\text {out }}(k)\right| \Psi\right\rangle$ are reasonable ${ }^{5}$ functions of $\mathbf{k}$ we can use the Riemann-Lebesgue Lemma for the integration over $\mathbf{k}$ and find that the right hand side vanishes in the limit $t \rightarrow \infty$ unless $m$ is equal to one of the particle masses. For $m=m_{i}$ one term is time independent and therefore survives in the limit. We get for $t \rightarrow+\infty$ if $m=m_{i}$

$$
\int_{x_{0}=t} Q(x) f(x) d^{3} x \rightarrow(2 \pi)^{+3 / 2} \sum_{i} \int d^{3} k F_{i}(\mathbf{k}) f(\mathbf{k}) a_{i}^{\dagger \text { out }}(\mathbf{k}) .
$$

4 For a definition see section II.

5 This will be justified in section 3 . 
The summation runs only over those particle types which have the same mass, namely $m$. Equation (9) is essentially the asymptotic relation used in $[1]^{6}$.

The generalization contained in (2) or (9) as compared to the original LSZ-relations [1] lies in the fact that they hold for any quasilocal operator, not only for the basic field operators. The only remnant of the choice of $Q$ is the function $F_{i}$ in (9). This allows a treatment of collision processes for "composite" particles or, from a more fundamental point of view, it allows a complete divorce between the number and types of fields in terms of which the theory is formulated and the number and types of particles which are described by the theory. Equation (9) has been derived in [2] and, for various special choices of $Q$, in [3], [4], [5]. In EKstern's method [3] $Q$ is chosen in such a way that the convergence in (9) becomes strong; in [4] and [5] the emphasis lies on the construction of a "strictly local" operator $Q$. In any case, (9) may be used to express the $S$-matrix element of any process in terms of vacuum expectation values of products (or time ordered products or retarded products) of quasilocal operators as discussed in [1], [6], [7], [8].

One remark should be added about the proof of the generalized LSZrelations from the basic postulates of Quantum Field Theory. The strongest result in this direction so far has been obtained by HEPP [10] using the techniques developed by RuELLE [9]. It proves the validity of (9) for matrix elements between states $\Psi$ and $\Phi$ where $\Phi$ may be arbitrary but $\Psi$ is restricted to a domain $D_{n}^{(+)}$(which will be described in section 3 of this paper). This proof will be reproduced in the course of our discussion in section 3 . It justifies most (though not all) of the customary applications of the LSZ-relations.

Our main objective in this paper, however, is to answer the question: What replaces equation (2) if $Q$ has vanishing matrix elements between the vacuum and the single particle states, i.e., if the functions $F_{i}$ and $G_{i}$ vanish? This question is of considerable interest because if particle $i$ is a Fermion or if it has an electric or baryonic charge then $F_{i}=G_{i}=0$ for every operator which belongs to the algebra of observables. In such a case one can, of course, treat the collision problems still in the framework of the LSZ-relations with the help of operators which do not belong to the algebra of observables (Fermi operators or other non gauge invariant quantities). Nevertheless we know that it must be possible to answer all questions of physical interest using only the algebra of observables [11]. In the present paper we shall show how collision cross

${ }^{6}$ In ref. [1] the quantity $i \int\left(Q(x) \frac{\partial f(x)}{\partial x_{0}}-\frac{\partial Q(x)}{\partial x_{0}} f(x)\right) d^{3} x$ is considered instead of $\int Q(x) \cdot f(x) d^{3} x$. This is, however, only a matter of formal elegance, irrelevant to our present discussion. 
sections can be obtained from the vacuum expectation values of products of observable quantities. The relevant asymptotic relation replacing (2) works with the matrix elements $\langle\mathbf{k} i|Q| \mathbf{k} i\rangle$. It is obtained by evaluating the next term appearing after the right hand side of (2) in an expansion of $\langle\Phi|Q(x)| \Psi\rangle$ into powers of $x_{0}^{-1}$. As mentioned before the term written down in (2) is of order $x_{0}^{-3 / 2}$. The next term, with which we are concerned here, is of order $x_{0}^{-3}$.

\section{Assumptions and notations}

The principal assumptions on which our argument is based are the principles of locality and invariance under the inhomogeneous Lorentz group together with some condition on the energy-momentum spectrum (spectral condition). These assumptions may either be expressed in terms of basic field operators (Wrghtman's axioms [12] or in terms of algebras of local observables [13], [14]). It is immaterial for the following results whether one uses the former or the latter framework. We shall adhere to the latter, in which one assumes that to each open region of finite extension in space-time there corresponds an algebra $R(\mathcal{O})$ of bounded operators in Hilbert space ${ }^{7}$. An operator from $R(\mathcal{O})$ may be regarded as the mathematical representative of a physical operation performable within $\mathcal{O}$. The algebra $\mathfrak{A}=\cup R(\mathcal{O})$ will be called the algebra of (all) local observables ${ }^{8}$. If $\mathcal{O}_{1}$ and $\mathcal{O}_{2}$ are two regions such that every point in $\mathcal{O}_{1}$ has a space-like distance from every point in $\mathscr{O}_{2}$ then every operator from $R\left(\mathcal{O}_{1}\right)$ shall commute with every operator from $R\left(\mathcal{O}_{2}\right)$. These statements summarize the principle of locality.

Lorentz invariance, the energy-momentum operators $P_{\mu}$, the mass operator $M=\sqrt{-P_{\mu}^{2}}$ are understood in the standard fashion (see e.g. [12]). Concerning the energy-momentum spectrum we assume that there is a unique vacuum state $|0\rangle$ and that all states orthogonal to it have positive energy and a mass which is not smaller than a certain value $m_{0} \neq 0$.

Unfortunately, in our present state of ignorance, we are forced to add a few other assumptions which are not as clearly understood in their relation to basic physical principles and which may in fact be partly redundant.

One complication arises from "superselection rules" or "gauge invariances". These imply that there exist local operations which do not belong to the algebra $R(\mathcal{O})$. Examples of such operations are the transfer

7 Within the present context there is no loss of generality if we assume that $R(\mathcal{O})$ is a von Neumann ring.

${ }^{8}$ The union is taken over all finitely extended open regions $\mathcal{O}$. Note that $\mathfrak{A}$ is not closed. Each element of $\mathfrak{A}$ is an operation in some finite region ("local" operation). Quasilocal and global operations will later be defined as limits of sequences in $\mathfrak{A}$ convergent in suitable topologies. 
of electric or baryonic charges from an observer to the observed system. These operations cannot be included in $R(\mathcal{O})$ because of a limitation in the superposition principle. If the original state of the system is described by the Hilbert space vector $\Psi$ and the state after this operation by $\Psi^{\prime}$ then the relative phase between $\Psi$ and $\Psi^{\prime}$ can have no physical significance since it depends on the choice of gauge. Therefore a linear combination $\alpha \Psi+\beta \Psi^{\prime}$ is in all respects equivalent to the mixture described by the statistical matrix $|\alpha|^{2}|\Psi\rangle\left\langle\left.\Psi|+| \beta\right|^{2} \mid \Psi^{\prime}\right\rangle\langle\Psi|$. We shall assume here the following simple and conventional model: There is a collection of Hilbert spaces $\mathscr{H}_{j}$ (the index $j$ corresponding to fixed values of the charge quantum numbers). The superposition principle holds unrestricted within each $\mathscr{H}_{j}$ and the algebra of observables $\mathfrak{A}$ transforms each $\mathscr{H}_{j}$ into itself. For formal convenience we work with the Hilbert space

$$
\mathscr{H}=\sum \mathscr{H}_{j}
$$

which is the direct sum of all the "coherent" spaces $\mathscr{H}_{j}$. Since the relative phases of vectors in different spaces $\mathscr{H}_{j}$ have no physical significance, the locality principle does not require that those local operations which change the charge quantum numbers commute at large space-like distances. We shall assume, that if the operator $Q_{\Delta}$ represents a local operation (in region $\mathcal{O}$ ) which changes the charges from $j$ to $j+\Delta$ then it is either a "Bose operator" or a "Fermi operator". The commutation relations between any pair of Bose operators $(B)$ and Fermi operators $(F)$ at space-like distances are:

$$
\begin{aligned}
& B B^{\prime}-B^{\prime} B=0 \\
& B F^{\prime}-F^{\prime} B=0 \\
& F F^{\prime}+F^{\prime} F=0
\end{aligned}
$$

where the primed and the unprimed operators refer respectively to regions $\mathcal{O}^{\prime}$ and $\mathcal{O}$ which are space-like to each other. Whether the operator $Q_{\Delta}$ is of Bose- or Fermi type shall depend only on $\Delta$.

Finally we shall assume that a complete particle interpretation is possible. This means that the Hilbert space $\mathscr{H}$ is spanned by the vectors $|c\rangle$ out where $c$ denotes an arbitrary particle configuration.

We denote the algebra of all operations in the region $\mathcal{O}$ by $\hat{R}(\mathcal{O})$. This includes those operations which change the charges. Correspondingly $\hat{\mathfrak{U}}=\hat{R}(\mathcal{O})$ is the algebra of all local operations. A dense set of vectors in the Hilbert space $\mathscr{H}$ is generated by application of $\hat{\mathfrak{A}}$ on the vacuum state $|o\rangle$ whereas the application of the algebra $\mathfrak{A}$ on the vacuum only gives a dense set of vectors in the zero charge sector $\mathscr{H}_{0}$.

Since the words "local", "quasilocal", "almost local" have been used in past publications by us and other authors in a variety of different meanings it may be good to specify exactly the definitions adopted in the 
present paper. We shall call "local" any operator which belongs to some $\hat{R}(\mathcal{O})$ (the symbol $\mathcal{O}$ always denotes a region of finite extension). Let $\mathscr{C}_{x, r}$ be the double cone in space-time whose base is a 3-dimensional sphere with radius $r$ and center at the space-time point $x$. Given an arbitrary operator $Q$ in the Hilbert space $\mathscr{H}$ we define the positive number

$$
d_{r}(Q)=\inf \|Q-B\|, \quad B \in R\left(\mathscr{C}_{0, r}\right)
$$

i.e., we look for the best approximation to $Q$ among all operators $B$ in a region of size $r$ around the origin. We call $Q$ "quasilocal of order $n$ " if

$$
\lim _{r \rightarrow \infty} r^{n} d_{r}(Q)=0 \text {. }
$$

Thus the completion of the algebra $\hat{\mathfrak{U}}$ in the norm topology leads to operators which are (at least) quasilocal or order zero. In the following we shall be principally concerned with quasilocal operators of infinite order. Therefore, if the order is not explicitly specified it will be understood that "quasilocal" means "quasilocal of infinite order".

\section{The extended asymptotic relations}

The following two basic lemmas have been proved by RuELLE [9]. The first concerns the asymptotic behavior of solutions of a KleinGordon equation.

Lemma 1. If

$f(x)=\int \tilde{f}(\mathbf{p}) e^{ \pm i p x} \frac{d^{3} p}{p_{0}} ; p x=\mathbf{p} \mathbf{x}-p_{0} x_{0} ; \quad p_{0}=\left(\mathbf{p}^{2}+m^{2}\right)^{1 / 2} ; \quad m \neq 0$, and ${ }^{9}$

$$
f(\mathbf{p}) \in \mathscr{S}
$$

let $\Sigma=\left\{\mathbf{p} / p_{0} \mid \mathbf{p} \in \operatorname{supp} f\right\}$ be the support of $f$ in velocity space and $U$ any open set containing $\Sigma$. Then

a) For $\mathbf{v} \notin U \quad|f(\mathbf{v} t, t)|<A_{N}(1+|v|)^{-N}|t|^{-N} ; N$ arbitrary

b) For $v \in U \quad|f(\mathrm{v} t, t)|<A^{\prime}|t|^{-3 / 2}$.

The constants $A_{N}, A^{\prime}$ can be chosen independent of $t$ and $\mathbf{v}$.

The second concerns the behavior of vacuum expectation values of products of quasilocal operators at large space-like distances and is a consequence of the principle of locality and the spectrum conditions as assumed in section 2 . One first makes a linked cluster (Ursell) decomposition of the functions $\left\langle o\left|Q\left(x_{1}\right) \ldots Q\left(x_{n}\right)\right| o\right\rangle$ :

$$
\begin{aligned}
\left\langle o\left|Q\left(x_{1}\right) \ldots Q\left(x_{n}\right)\right| o\right\rangle & \\
& =\Sigma\left\langle Q\left(x_{1}^{(1)}\right) \ldots Q\left(x_{n_{1}}^{(1)}\right)\right\rangle_{T}\left\langle Q\left(x_{1}^{(2)} \ldots Q\left(x_{n}^{(2)}\right)\right\rangle_{T} \ldots\langle\rangle_{T}\right.
\end{aligned}
$$

$9 \mathscr{S}$ is the class of infinitely differentiable functions which vanish faster than any power at infinity. 
where $x_{1}^{(1)} \ldots x_{n_{k}}^{(k)}$ are a partition of the $n$ points $x_{1} \ldots x_{n}$ into $k$ "clusters" and the summation runs over all such partitions. The operator $Q(x)$ is, of course, understood as in Eq. (4). The system of equations (14) for all values of $n$ defines the "truncated expectation values" $\left\langle Q\left(x_{j}\right) \ldots Q\left(x_{l}\right)\right\rangle_{T}$.

Lemma 2. Let $Q_{j}$ be quasilocal and

$$
\delta=\max _{i, j}\left|\left(x_{i}\right)_{0}-\left(x_{i}\right)_{0}\right| ; \quad R=\max _{i, j}\left|\mathbf{x}_{i}-\mathbf{x}_{j}\right| .
$$

Then, varying the $x_{j}$ with the only restriction that $\delta$ remains smaller than a fixed limit $\bar{\delta}$ one has

$$
\left|\left\langle Q_{1}\left(x_{1}\right) \ldots Q_{n}\left(x_{n}\right)\right\rangle_{T}\right|<A_{\dot{N}} R^{-N}
$$

where $N$ is arbitrary, $A_{N}$ independent of the configuration $x_{j}$.

Further we shall use

Lemma 3. For each particle type $i$ one can construct a quasilocal operation $q_{i}$ which creates a one particle state of this type from the vaccum:

$$
q_{i}|o\rangle=\int \varphi(\mathbf{p})|\mathbf{p} i\rangle d^{3} p .
$$

The wave function

$$
\varphi_{i}(\mathbf{p})=\left\langle\mathbf{p} i\left|q_{i}\right| 0\right\rangle
$$

is infinitely often differentiable and $q_{i}$ may be chosen so that $\varphi_{i}(\mathbf{p})$ does not vanish anywhere.

Although this lemma is part of the folk lore it may be helpful to indicate the proof of its various assertions. In the first place there must be some local operator $Q$ for which the matrix element $\langle p i|Q| 0\rangle \neq 0$ for almost all $\mathbf{p}$ in any finite preassigned region of $p$-space. Otherwise we could not generate a dense set in $\mathscr{H}$ by $\hat{\mathfrak{U}}|o\rangle$. Starting with such an operator we can form a quasilocal operator $q=\int Q(x) f(x) d^{4} x$, choosing a test function $f$ which decreases faster than any power at large distances in 4-dimensional $x$-space and whose Fourier transform $f(p)$ has support only in a small neighborhood of the mass hyperboloid of particle $i$. This operator will then satisfy (16) with

$$
\varphi(\mathbf{p})=\tilde{f}\left(\mathbf{p}, \sqrt{\left.p^{2}+m_{i}^{2}\right)}\langle\mathbf{p} i|Q| o\rangle .\right.
$$

Furthermore, if $Q^{\prime}$ is any other quasilocal operator we know from lemma 2 that

$$
\left\langle 0\left|Q^{\prime \dagger}(\mathbf{x}) q\right| 0\right\rangle=\int\left\langle\mathbf{p}, i\left|Q^{\prime}\right| 0\right\rangle *\langle\mathbf{p} i|Q| o\rangle f\left(\mathbf{p}, \sqrt{\left.\mathbf{p}^{2}+m_{i}^{2}\right)} e^{+i \mathbf{p x}} d^{3} p\right.
$$

must decrease faster than any power of $|\mathbf{x}|$ or in other words that

$$
\left\langle\mathbf{p} i\left|Q^{\prime}\right| o\right\rangle^{*}\langle\mathbf{p} i|Q| o\rangle f\left(\mathbf{p}, \sqrt{\left.\mathbf{p}^{2}+m_{i}^{2}\right)}\right.
$$

must be infinitely often differentiable with respect to $\mathbf{p}$ for any pair of quasilocal operators $Q, Q^{\prime}$ and any class- $\mathscr{S}$-function $F(\mathbf{p})=f\left(\mathbf{p}, \sqrt{\left.p^{2}+m_{i}^{2}\right)}\right.$. We conclude that $\langle\mathbf{p} i|Q| o\rangle$ can differ from an infinitely differentiable 
function at most by a phase factor $e^{i \Delta(\mathbf{p})}$ and that furthermore the difference $\Delta_{Q}(\mathbf{p})-\Delta_{Q^{\prime}}(\mathbf{p})$ for any quasilocal pair $Q, Q^{\prime}$ must again be infinitely differentiable. On the other hand we know that any finite Lorentz transform of a quasilocal operator is again quasilocal. Choosing $Q^{\prime}=U(\Lambda) Q U^{-1}(\Lambda)$ and letting $\Lambda$ tend towards the identity we find that $\frac{\partial \Delta_{Q}}{\partial p i}$ is infinitely differentiable and hence the same holds for $\Delta_{Q}$. This demonstrates the smoothness of $\varphi_{i}(\mathbf{p})$ claimed in the lemma. To make $\varphi_{i}(\mathbf{p})$ nonvanishing everywhere we can use again Lorentz transformations. If $\phi_{i}$ vanishes at $\mathbf{p}$ then we can always find a Lorentz transformation $\Lambda$ such that $\left\langle\mathbf{p} i\left|U(\Lambda) q_{i} U^{-1}(\Lambda)\right| 0\right\rangle$ does not vanish there. We take then, instead of $q_{i}$ the operator $q_{i}^{\prime}=\int U(\Lambda) q_{i} U^{-1}(\Lambda) d \mu(\Lambda)$ which is again quasilocal as long as we choose a measure $\mu(\Lambda)$ which decreases sufficiently fast for large Lorentz transformations. For suitable choices of $\mu$ the function $\left\langle\mathbf{p} i\left|q_{i}^{\prime}\right| 0\right\rangle$ will be non zero everywhere.

According to these three lemmas one can now use the method of ref. [2] to construct the state vectors describing arbitrary incoming or outgoing particle configurations:

$$
\begin{aligned}
&|\alpha i, \beta j \ldots\rangle^{\text {in }}=\lim _{t \rightarrow-\infty} \Psi_{\alpha i, \beta j \ldots}(t) \\
&|\alpha i, \beta j \ldots\rangle^{\text {out }}=\lim _{t \rightarrow+\infty} \Psi_{\alpha i, \beta j \ldots}(t) \\
& \Psi_{\alpha i, \beta j}(t)=q_{i}^{\alpha}(t) q_{j}^{\beta}(t) \ldots|o\rangle \\
& q_{i}^{\alpha}(t)=\int g_{i}^{\alpha}(\mathbf{x}, t) q_{i}(\mathbf{x}, t) d^{3} x ; \\
& g_{i}^{\alpha}(x)=(2 \pi)^{-3} \int \frac{\psi_{\alpha i}(p)}{\varphi_{i}(p)} e^{i\left(\mathbf{p} \mathbf{x}-\sqrt{\left.\mathbf{p}^{2}+m_{i}^{2} t\right)}\right.} d^{3} p .
\end{aligned}
$$

Here $\psi_{\alpha i}(\mathbf{p})$ is the momentum space wave function of particle $i$ in the state we want to construct; $q_{i}$ and $\varphi_{i}$ are as defined in lemma 3 . In the following we shall frequently abbreviate the index combination $\alpha i, \beta j \ldots$ by a single label $\lambda$ and write $\Psi_{\lambda}^{\text {in }}$ instead of $|\lambda\rangle^{\text {in }}$.

The rate of convergence in (19), (20) can be investigated with the help of the three lemmas. One finds

Theorem 1. a) If the single particle wave functions $\psi_{\alpha i}(\mathbf{p}), \psi_{\beta j} \ldots$ belong to class $\mathscr{D}^{10}$ then

$$
\left\|\Psi_{\lambda}^{\text {out }}-\Psi_{\lambda}(t)\right\|<A t^{-1 / 2} \text { for } t>0 .
$$

b) If in addition the $\Psi_{\alpha i}, \Psi_{\beta j} \ldots$ have disjoint support in velocity space (see lemma 1) then

$$
\left\|\Psi_{\lambda}^{\text {out }}-\Psi_{\lambda}(t)\right\|<A_{N} t^{-N} \quad \text { for } \quad t>0
$$

and any positive $N$.

Corresponding statements are true for $t<0$ with $\Psi^{\text {in }}$ replacing $\Psi^{\text {out }}$.

10 This is the class of infinitely differentiable functions with compact support. 
The proof of a) is given in [9]. While b) is not explicitly pointed out there it follows easily from the same argument. This observation has been made also in [10].

Part b) of this theorem will be of crucial importance for our further discussion. We define the domains $D^{(+)}$and $D_{n}^{(+)}$in Hilbert space as the collection of those vectors which can be obtained by linear combinations of a finite number of $\Psi^{\text {out }}$ where the particle configurations $\lambda$ satisfy respectively the assumptions of theorem $1 \mathrm{a}$ ) or $1 \mathrm{~b}$ ). Roughly speaking $D^{(+)}$contains outgoing particle states with class- $\mathscr{D}$-wave functions, $D_{n}^{(+)}$ contains only those states of $D^{(+)}$in which no pair of particles can have the same velocity. In a corresponding way we define $D^{(-)}$and $D_{n}^{(-)}$(replacing "out" by "in").

Lemma 4.

$$
D^{(+)}, D^{(-)}, D_{n}^{(-)}, D_{n}^{(-)} \text {are dense in } \mathscr{H} .
$$

Since we have assumed that a complete particle interpretation is possible, i.e., that the $\Psi_{\lambda}^{\text {out }}$ as well as the $\Psi_{\lambda}^{\text {in }}$ span $\mathscr{H}$, the proof of lemma 4 is reduced to the demonstration that an arbitrary square integrable function $f\left(\mathbf{p}_{1} \ldots \mathbf{p}_{n}\right)$ can be approximated in the mean by finite linear combinations of the form $\Sigma c_{\alpha \beta} \ldots f_{\alpha}\left(\mathbf{p}_{1}\right) f_{\beta}\left(\mathbf{p}_{2}\right) \ldots f_{\varrho}\left(\mathbf{p}_{n}\right)$ with the functions $f_{\alpha}, f_{\beta} \ldots$ belonging to class $\mathscr{D}$ and having disjoint supports. This is easily demonstrated by making an increasingly fine cell division in p-space and choosing the $f_{\alpha}$ as (smoothed out) characteristic functions of the various cells.

We are now ready to state our main theorems:

Theorem 2. If $Q$ is quasilocal and $\Psi$ and $\Phi$ are in $D_{n}^{(+)}$, then

$$
\begin{aligned}
\langle\Phi|Q(x)| \Psi\rangle= & \langle o|Q| o\rangle\langle\Phi \mid \Psi\rangle+\sum_{i} \int d^{3} p\langle\mathbf{p} i|Q(x)| 0\rangle\left\langle\Phi\left|a_{i}^{\text {¡out }}(\mathbf{p})\right| \Psi\right\rangle \\
& +\langle 0|Q(x)| \mathbf{p} i\rangle\left\langle\Phi\left|a_{i}^{\text {out }}(\mathbf{p})\right| \Psi\right\rangle \\
+ & \sum_{i j} \int d^{3} p d^{3} p^{\prime}\left\langle\mathbf{p}^{\prime} j|Q(x)| \mathbf{p} i\right\rangle\left\langle\Phi\left|a_{j}^{\text {¡out }}\left(\mathbf{p}^{\prime}\right) a_{i}^{\text {out }}(\mathbf{p})\right| \Psi\right\rangle+R
\end{aligned}
$$

with

$$
|R|<A_{N} x_{0}^{-N} \text { for } \quad x_{0}>0
$$

and arbitrary positive $N$. The constant $A_{N}$ depends on $Q, \Psi$ and $\Phi$ but not on $x$. The analogous result holds for $x_{0}<0$ if $\Psi, \Phi$ are in $D_{n}^{(-)}$and the superscript "out" is replaced by "in".

Note that the first term on the right hand side is independent of $x$, the next term (LSZ-term, see introduction) decreases like $x_{0}^{-3 / 2}$ asymptotically, the third term decreases like $x_{0}^{-3}$ and the remainder $R$ decreases stronger than any power.

Proof of theorem 2. Consider $\Psi=\Psi_{\lambda}^{\text {out }}, \Phi=\Phi_{\lambda^{\prime}}^{\text {out }}$ and replace them by $\Psi_{\lambda}(t), \Psi_{\lambda^{\prime}}(t)$, choosing $t=x_{0}$. According to theorem $1 \mathrm{~b}$ the mistake made in this replacement can be included in $R$. We are then left with the eva- 
luation of an equal time vacuum expectation value

$$
\begin{aligned}
& \left\langle o\left|\ldots q_{j \prime}^{\dagger \beta^{\prime}}(t) q_{i}^{\dagger \alpha^{\prime}}(t) Q(\mathbf{x}, t) q_{i}^{\alpha}(t) q_{j}^{\beta}(t) \ldots\right| o\right\rangle \\
& \quad=\int \Pi d^{3} y_{k} d^{3} z_{l} g_{k}^{*}\left(\mathbf{y}_{k}, t\right) g_{l}\left(\mathbf{z}_{l}, t\right)\langle o| \ldots q^{\dagger}\left(\mathbf{y}_{k}, t\right) \ldots Q(\mathbf{x}, t) \ldots \\
& q\left(\mathbf{z}_{l}, t\right) \ldots|o\rangle
\end{aligned}
$$

to which we can apply lemma 1 and 2 . We have omitted detailed indices on the right hand side to make the structure of the expression more transparent. Making an Ursell decomposition of the vacuum expectation value one gets sums of products of truncated expectation values of various subsets (clusters) of the $q_{k}^{\dagger}(t), q_{l}(t), Q(x)$. The lemmas 1,2 tell us that any such term will decrease faster than any power of $t$ unless all the supports in velocity space of the wave functions $g_{k}^{*}, g_{l}$ which appear in the same cluster have a non-empty intersection. Since $\Psi^{\text {out }}$ as well as $\Phi_{\lambda^{\prime}}^{\text {out }}$ belong to $D_{n}^{(+)}$the supports of any pair of wave functions entering into $\Psi_{\lambda^{\prime}}^{\text {out }}$ are disjoint and the same is true for any pair entering into $\Phi_{\lambda^{\prime}}^{\text {out }}$. Thus we need consider only the truncated functions which involve at most one $q_{l}(t)$ and at most one $q_{k}^{*}(t)$. This leaves the following four types of truncated functions involving $Q$ :

$$
\left.\langle Q(x)\rangle ;\left\langle Q(x) q_{l}(t)\right\rangle_{T} ;\left\langle q_{k}^{\dagger}(t) Q(x)\right\rangle_{T} ;\left\langle q_{k}^{\dagger}(t) Q(x) q_{l}(t)\right)\right\rangle_{T} .
$$

Each of these expressions is multiplied with the vacuum expectation value of the product of remaining operators $q^{\dagger}, q$. These coefficients are respectively (neglecting quantities which vanish like $t^{-N}$ )

$$
\begin{aligned}
\left\langle\Phi_{\lambda^{\prime}}^{\text {out }}, \Psi_{\lambda}^{\text {out }}\right\rangle ;\left\langle\Phi_{\lambda^{\prime}}^{\text {out }}\left|a_{l}^{\text {out }}\right| \Psi_{\chi}^{\text {out }}\right\rangle ; & \\
& \left\langle\Phi_{\lambda^{\prime}}^{\text {out }}\left|a_{k}^{\text {out }}\right| \Psi_{\lambda}^{\text {out }}\right\rangle ;\left\langle\Phi_{\lambda^{\prime}}^{\text {out }}\left|a_{\hbar}^{\text {out }} a_{l}^{\text {out }}\right| \Psi_{\lambda}^{\text {out }}\right\rangle
\end{aligned}
$$

with the abbreviation $(l=i, \alpha)$

$$
a_{l}^{\text {out }}=\int\left[\varphi_{i}^{\alpha}(\mathbf{p})\right]^{-1} a_{i}(\mathbf{p}) d^{3} p \quad \text { etc. }
$$

This result coincides with the claim of theorem 2 for the special case $\Phi=\Phi_{\lambda^{\prime}}^{\text {out }}, \Psi=\Psi_{\lambda}^{\text {out }}$. Since a general vector in $D_{n}^{(+)}$is a finite linear combination of vectors $\Psi_{\lambda}^{\text {out }}$ we have thereby proved the theorem.

In most applications one would like to use formula (23) for $\Phi$ arbitrary and only $\Psi$ restricted to $D_{n}^{(+)}$. This can be justified to some extent by means of the following lemmas.

Lemma 5. Let $Q$ be quasilocal and $\langle o|Q| 0\rangle=0$. Define

$$
Q(h, t)=\int Q(\mathrm{v} t, t) h(\mathrm{v}) d^{3} v
$$

where $h$ is a bounded function. Then

$$
t^{3 / 2}\|Q(h, t) \Psi\|<A \quad \text { for } \Psi \in D_{n}^{(+)} \text {and } t>0 .
$$

Lemma 6. Let $C$ be quasilocal and $C|o\rangle=C^{\dagger}|o\rangle=0, C(h, t)$ defined as in lemma 5. Then

$$
t^{3}\|C(h, t) \Psi\|<A \quad \text { for } \quad \Psi \in D_{n}^{(+)} \quad \text { and } \quad t>0 .
$$


Proof. We have to show that $t^{3}\left\langle\Psi\left|Q^{\dagger}(h, t) Q(h, t)\right| \Psi\right\rangle$ and $t^{6}\left\langle\Psi\left|C^{\dagger}(h, t) C(h, t)\right| \Psi\right\rangle$ are bounded for positive $t$. For this purpose it is again sufficient to replace $\Psi$ by some $\Psi_{\lambda}(t)$. The method used in the proof of theorem 2 can thus be applied and yields in a straightforward way the desired result. This gives us

Theorem 3. The generalized $L S Z$ relation (9) is valid for matrix elements between states $\Psi, \Phi$ as long as $\Psi \in D_{n}^{(+)}$while $\Phi$ may be arbitrary.

Theorem 4. If $C$ is as in lemma 6 and $\left\langle\mathbf{p}^{\prime} j|C| \mathbf{p} i\right\rangle$ is differentiable with respect to $p$ and $p^{\prime}$ then for $\Psi \in D_{n}^{(+)}$and $\Phi$ arbitrary one has

$\lim _{t \rightarrow+\infty}\left\langle\Phi\left|t^{3} C(h, t)\right| \Psi\right\rangle=\Sigma^{\prime} \int d^{3} p \Gamma_{i j}(\mathbf{p})\left\langle\Phi\left|a_{j}^{\dagger \text { out }}(\mathbf{p}) a_{i}^{\text {out }}(\mathbf{p})\right| \Psi\right\rangle h\left(\mathbf{v}_{i}\right)$

with

$$
\begin{aligned}
\Gamma_{i j}(\mathbf{p}) & =8 \pi^{3}\langle\mathbf{p} j|C(0)| \mathbf{p} i), \\
\mathbf{v}_{i} & =\left(\mathbf{p}^{2}+m_{i}^{2}\right)^{-1 / 2} \mathbf{p} .
\end{aligned}
$$

The symbol $\Sigma^{\prime}$ means that the summation includes only those pairs of particles with $m_{i}=m_{j}$.

Proof. We note that for large $t$ the operator $\int_{x_{0}=t} Q(x) f(x) d^{3} x$ which appears on the left hand side of (9) is of the form $t^{3 / 2} Q(h, t)$. Therefore it is sufficient to prove theorems 3 and 4 for the special case in which both $\Phi$ and $\Psi$ belong to $D_{n}^{(+)}$. The extension to arbitrary $\Phi$ follows then from lemmas 5 and 6.

If $\Phi$ and $\Psi$ are in $D_{n}^{(+)}$we apply theorem 2 . The step from there to equation (9) has already been described in the introduction.

Concerning theorem 4 we find for the left hand side of (28) from theorem 2 and application of translational invariance, the expression

$$
\begin{aligned}
t^{3} \int d^{3} v d^{3} p^{\prime} & d^{3} p h(v) \sum_{i j}\left\langle\mathbf{p}^{\prime} j|C(0)| \mathbf{p}_{i}\right\rangle \exp i\left[\left(\mathbf{p}-\mathbf{p}^{\prime}\right) \mathbf{v}-\right. \\
& \left.-\left(\sqrt{\mathbf{p}^{2}+m_{i}^{2}}-\sqrt{\mathbf{p}^{\prime 2}+m_{j}^{2}}\right)\right]\left\langle\Phi\left|a_{j}^{\dagger \text { out }}\left(\mathbf{p}^{\prime}\right) a_{i}^{\text {out }}(\mathbf{p})\right| \Psi\right\rangle .
\end{aligned}
$$

Since the integrand is a differentiable, fast decreasing function of $\mathbf{p}$ and $\mathbf{p}^{\prime}$ one can evaluate the integrals with respect to $\mathbf{p}$ and $\mathbf{p}^{\prime}$ by the method of stationary phase. The phase is stationary with respect to variations of $\mathbf{p}$ and $\mathbf{p}^{\prime}$ at

$$
\mathbf{p}\left(\mathbf{p}^{2}+m_{i}^{2}\right)^{-1 / 2}=\mathbf{p}^{\prime}\left(\mathbf{p}^{\prime 2}+m_{j}^{2}\right)^{-1 / 2}=\mathbf{v} .
$$

If $m_{i} \neq m_{j}$ the phase is not stationary there with respect to variations of $\mathbf{v}$ and hence the $\mathbf{v}$-integration will then produce a stronger decrease with $t$ than in the case $m_{i}=m_{j}$. Thus we may omit the terms in the summation over $i$ and $j$ for which $m_{i} \neq m_{j}$. In other words we may replace $\Sigma$ by $\Sigma^{\prime}$ as described in the theorem. For $m_{i}=m_{j}$ we have at the stationary point

$$
\mathbf{p}=\mathbf{p}^{\prime}=m_{i} \mathbf{v} / \sqrt{1-\mathbf{v}^{2}}
$$


and the expression (30) reduces to

$$
\int d^{3} v \sum_{i j}^{\prime} \frac{8 \pi^{3} p_{0}^{5}}{m_{i}^{2}} h(\mathrm{v})\left\langle\Phi\left|a_{j}^{\dagger \text { out }}(\mathbf{p}) a_{i}^{\text {out }}(\mathbf{p})\right| \Psi\right\rangle\langle\mathbf{p} j|C(0)| \mathbf{p} i\rangle
$$

where $\mathbf{p}$ is related to $\mathbf{v}$ by (31). Changing the integration variable from $\mathbf{v}$ to $\mathbf{p}$ (the Jacobian is just $\left.\frac{m^{2}}{p_{0}^{5}}\right)$ we obtain the right hand side of (28).

One checks further that the difference between the left and the right hand side of (28) vanishes stronger than $t^{-1}$ in the limit if one applies the Riemann-Lebesque lemma to the remainder term in the stationary phase method.

It is easy to generalize theorem 4 to

Theorem 5. If the $C_{\alpha}, \Psi$ and $\Phi$ are as in theorem 4 and if $h_{\alpha}(\mathrm{v})$ are class-S $\mathscr{S}$-functions with non overlapping support then

$\lim \left\langle\Phi\left|t^{3 n} C_{1}\left(h_{1}, t\right) \ldots C_{n}\left(h_{n}, t\right)\right| \Psi\right\rangle$

$=\Sigma \int d^{3} p_{1} \ldots d^{3} p_{n} \Gamma_{i_{1} j_{1}}\left(p_{1}\right) \Gamma_{i_{n} j_{n}}\left(\mathbf{p}_{n}\right)\left\langle\Phi\left|\varrho_{i_{1} j_{1}}^{\text {out }}\left(\mathbf{p}_{1}\right) \ldots \varrho_{i_{n} j_{n}}^{\text {out }}\left(\mathbf{p}_{n}\right)\right| \Psi\right\rangle \Pi h_{k}\left(\mathbf{v}_{k}\right)$

with

$$
\varrho_{i j}^{\text {out }}(\mathbf{p})=a_{j}^{\text {† out }}(\mathbf{p}) a_{i}^{\text {out }}(\mathbf{p}) .
$$

\section{Cross sections}

The physical meaning of Eq. (33) is rather simple. The operators were restricted by the conditions

i) $C^{\dagger}=C$

ii) $C|o\rangle=o$

iii) $C$ is quasilocal.

The first two conditions tell us that $C$ is an observable which has the vacuum state as an eigenstate to eigenvalue zero. The third condition means that $C(x)$ is a measurement in the neighborhood of the spacetime point $x$. Therefore a nonvanishing expectation value of $C(x)$ in a state $\Psi$ indicates that the state $\Psi$ has a local deviation from the vacuum in the neighborhood of the point $x$. In other words, $C(x)$ can be considered as representing some sort of a detector placed at the mean position $x$. The product $C\left(x_{1}\right) \ldots C\left(x_{n}\right)$ for equal times and large spatial separation of the points is the mathematical representative of a coincidence arrangement of $n$ such detectors. If we let the time $t=x_{10}=x_{20}$ $=x_{n 0}$ become larger and larger, i.e. if we place the coincidence arrangement further and further from the source which prepares the state (the target in a typical collision experiment) then the probability that one detector registers a single particle decreases like $t^{-3}$ (by elementary geometry) whereas the probability that any other local excitation (more complicated than a single particle) hits the detector decreases faster than $t^{-3}$. Thus in the limit $t \rightarrow \infty$ each detector becomes a particle 
counter. The functions $\Gamma_{i j}(\mathbf{p})$ appearing in (33) and defined in Eq. (29) may be called the sensitivity matrix of the detector. Clearly, the diagonal element $\Gamma_{i i}(\mathbf{p})$ is a measure of the sensitivity for a particle of type $i$ and momentum p. Since there is only a finite number of particle types (including spin orientations) to one value of the mass, it is easy to choose $C$ so that the matrix $\Gamma$ is diagonal i.e.

$$
\Gamma_{i j}(\mathbf{p})=\delta_{i j} \Gamma_{i}(\mathbf{p}) \text {. }
$$

These sensitivity functions are then the only relevant pieces of information we need to know about $C$ in asymptotic measurements. In the following we shall assume for convenience that such a choice of $C$ has been made.

It is worthwhile at this point to pursue the correspondence between physical apparatus and mathematical objects a little further. NIELs BoHR, on a skiing trip with friends, once compared the progress of physics to the problem they faced that night in their chalet: to get the dishes clean in an environment where everything was dirty. The mathematical physicist is apt to dislike this comparison. In our context he would prefer to deal with idealized detectors, objects which are uniquely specified both within the mathematical frame and by their construction manual. However, as NIELs BoHR's analogy points out, this is neither necessary nor would it be a fair description of what is really done. On the experimental side there is an infinite variety of ways in which a detector may be built. The development of an apparatus suitable for a particular purpose (highly selective sensitivities) is a lengthy process involving trial and error to a large degree. Exactly the same situation prevails in the mathematical description as we have pictured it. The conditions (i), (ii), (iii) are so weak that there is no problem at all in finding any number of operators $C$ which might serve. Most of them will correspond to poor detectors. Improvement again is a matter of intelligent trial.

To summarize: The physical interpretation of the mathematical scheme is provided in a minimal fashion, involving only geometric concepts. We identify in the mathematical frame the representatives of the geometric invariance group (inhomogeneous Lorentz group) and we identify the class of all physical operations which can be performed in a space-time region $\mathcal{O}$. No attempt is made to identify individual operators from $R(\mathcal{O})$ with specific pieces of hardware.

After this digression, we may discuss the use of Eq. (33). By letting the functions $h_{k}$ approach $\delta$-functions we can, for any arbitrary state vector $\Psi$, obtain the expectation value

$$
\left\langle\Psi\left|\varrho_{i_{1}}^{\text {out }}\left(\mathbf{p}_{1}\right) \ldots \varrho_{i_{n}}^{\text {out }}\left(\mathbf{p}_{n}\right)\right| \Psi\right\rangle
$$

expressed in terms of time limits of expectation values of products of quasilocal operators. The quantity (36) is the probability density in 
$p$-space for finding $n$ outgoing particles with momenta $p_{1} \ldots p_{n}$ plus an arbitrary number of unspecified additional outgoing particles in the state $\Psi$. The probability density for finding exactly $n$ outgoing particles with specified momenta in the state $\Psi$ can be obtained either by subtracting from (36) the probability for higher particle numbers or, more conveniently by the following method: Usually one is dealing with states $\Psi$ such that the energy-momentum is known to lie in a neighborhood $\Delta$ which is so small that the difference between any two momentum vectors in $\Delta$ lies below the lowest mass hyperboloid. In this case it sufficies to consider (36) for such momentum configurations that $\Sigma p_{k}$ lies in $\Delta$ and then (36) gives directly the probability density for the $n$-particle configuration.

While the relation (33) is useful in analyzing a given state in terms of its outgoing (or incoming) particle configurations, it does not provide a convenient method for constructing a state with a specified initial particle configuration. This corresponds to the obvious fact that detectors are not very useful instruments in the preparation of a state. For this reason, we are not able to give here a compact expression for cross sections in terms of vacuum expectation values of "observables" (gauge invariant quantities). From the point of view of simplicity and elegance, the algorithm of the LSZ-reduction formulas (using non gauge invariant quantities) is much superior to (33). On the other hand it is clear that by a combination of energy-momentum restrictions on the state and monitoring experiments, using the analogue of (33) for $t \rightarrow-\infty$, one can determine the initial particle configuration. Therefore one can arrive at expressions for cross sections using only the algebra of observables. But the procedure is inelegant and tedious.

We would like to draw the following conclusions:

1. From the point of view of principle it appears that a formulation of the theory using only "observables" (i.e., no Fermi fields, no charge transfer operators) is possible.

2. From a practical point of view the use of "non-observable" fields is certainly preferable at least for the purpose of constructing a state vector which represents a specified configuration of incoming particles. Formula (33) gives then a simple expression for the scattering cross sections (though not for the $S$-matrix).

3. Theorem 2 is sometimes useful in analyzing the behaviour of Green's functions for large time differences. One recent example, in which the term with $\varrho^{\text {out }}$ in (23) is important, is the derivation of the AdlerWeissberger relations with the Fubini-Furlan technique (see [15], [16]). While the essential parts of the discussion in [15] are correct, the paradoxies in the appendix disappear if proper use is made of our theorem 2 . 
Acknowledgement. We want to thank Dr. D. W. Robinson for discussions and the National Science Foundation for financial support.

\section{References}

[1] Lehmann, H., K. Symanzik, and W. Zimmermann: Nuovo Cimento 1, 425 (1955).

[2] HAAG, R.: Phys. Rev. 112, 669 (1958).

[3] Ekstein, H.: Nuovo Cimento 4, 1017 (1956).

[4] Zimmermann, W.: Nuovo Cimento 10, 597 (1958).

[5] NishiJima, K.: Phys. Rev. 111, 995 (1958).

[6] - Progr. Theoret. Phys. 17, 765 (1957).

[7] Lemmann, H., K. Symanzik, and W. Zimmermans: Nuovo Cimento 6, 319 (1957).

[8] Glaser, V., H. Lehmann, and W. Zimmermann: Nuovo Cimento 6, 1122 (1957).

[9] Ruelle, D.: Helv. Phys. Acta 35, 162 (1962).

[10] Hepp, K.: Commun. Math. Phys. 1, 301 (1965).

[11] HaAg, R., and D. Kastler: J. Math. Phys. 5, 848 (1964).

[12] Wightman, A. S.: Paris Lectures, 1956 (unpublished).

[13] HaAG, R., and B. Schroer: J. Math. Phys. 3, 248 (1962).

[14] Araki, H.: Zurich Lectures, 1962 (unpublished).

[15] Окиво, S.: Nuovo Cimento 41, 586 (1966).

[16] Schroer, B., and P. Stichel: Commun. Math. Phys. 3, 258 (1966). 